\title{
Network Coding Schemes for Device-to-Device Communications Based Relaying for Cellular Coverage Extension
}

\author{
Andrea Abrardo*†, Gábor Fodor ${ }^{\sharp \sharp}$, Besmir Tola* \\ * Department of Information Engineering and Mathematics, University of Siena, Italy. E-mail: abrardo@dii.unisi.it \\ ${ }^{\dagger}$ CNIT Research Unit of Siena, Italy. \\ ¥ Ericsson Research, Stockholm, Sweden. E-mail: gabor. fodor@ericsson.com \\ \# Royal Institute of Technology, Stockholm, Sweden. E-mail: gaborf@kth.se
}

\begin{abstract}
Although network assisted device-to-device (D2D) communications is known to improve the spectral and energy efficiency of proximal communications, its performance is less understood when employed to extend the coverage of cellular networks. In this paper, we study the performance of D2D based range extension in terms of sum rate and power efficiency when a relaying user equipment (UE) helps to improve the coverage for cell-edge UEs. In our design, the relaying UE has own traffic to transmit and receive to/from the cellular base station (BS) and can operate either in amplify-and-forward (AF) or decodeand-forward (DF) modes and can make use of either digital or analogue (PHY layer) network coding. In this rather general setting, we propose mode selection, resource allocation and power control schemes and study their performance by means of system simulations. We find that the performance of the DF scheme with network coding is superior both to the traditional cellular and the AF based relaying schemes, including AF with two-slot or three-slot PHY layer network coding.
\end{abstract}

\section{INTRODUCTION}

Although the ideas of integrating multi-hop (MH) and relaying systems into cellular networks are not new [1], [2], the advantages of Device-to-Device (D2D) communications in cellular spectrum have been identified and analyzed only recently [3], [4]. Recent results suggest that D2D communications can increase the spectral and energy efficiency by taking advantage of the proximity, reuse and hop gains when radio resources are properly allocated to the cellular and D2D layers [5]. Using cellular spectrum is known to have the advantages of a managed interference environment compared with relying on unlicensed bands [6], [3], [4].

Another line of research suggests that relay-assisted $\mathrm{MH}$ communications, including mobile relays and relay-assisted D2D communications can not only enhance the achievable transmission capacity, but can also improve the coverage of cellular networks [4], [7], [8], [9], [10], [11]. Specifically, in our recent works, we found that network assisted two-hop D2D communications enhances the coverage, improves the energy efficiency of cellular networks and can also be useful in providing national security and public safety (NSPS) services

\footnotetext{
${ }^{1}$ The work of G. Fodor has been partially performed in the framework of the FP7 project ITC 317669 METIS. G. Fodor has also been supported by the Swedish Foundation for Strategic Research Strategic Mobility SM13-0008 Matthew Project.
}

[12], [13]. Indeed, as illustrated in Figure 1, when a user equipment (UE2) is willing to provide relaying assistance to a cell edge UE (UE1 of Figure 1) - in addition to managing its own traffic -, the service quality of UE1 can largely improve provided that the proper communication mode - cellular mode or relay mode - is selected for UE1.

\section{Cellular and Relay Modes for UE-to-BS Communications}

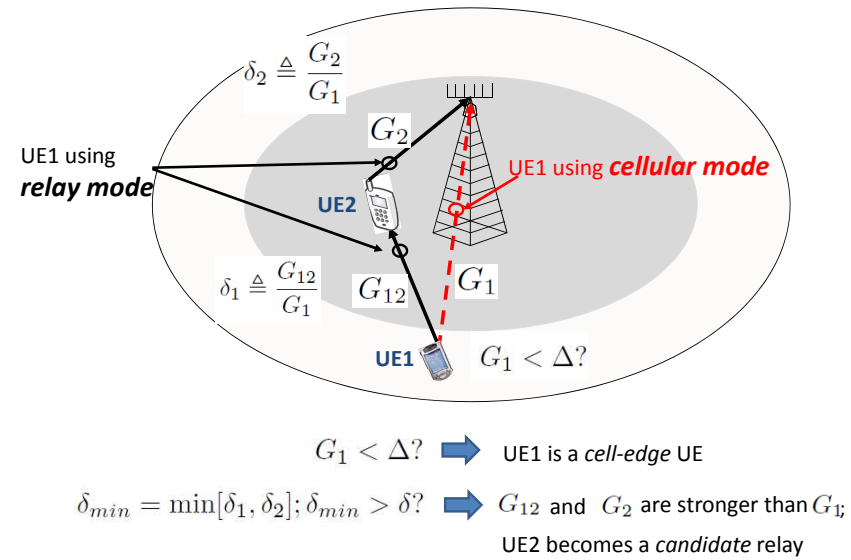

Fig. 1. D2D communication modes: A cell-edge UE (here: UE1) can attempt to communicate with a cellular BS either in cellular mode or in relay mode using D2D communications and the assistance of a helper UE (here: UE2). In relay mode, using D2D communications, the resources allocated to the UE1UE2 link can be reused by either cellular links or other D2D links within the coverage area of the cell. We refer to the UE2 that for a given UE1 maximizes $\delta_{\min }$ as the 'best' relay for UE1.

As it is intuitively clear from Figure 1, when D2D communications is used for cellular range extension, the following inherently inter-twined radio resource management tasks must be addressed:

- Mode Selection (MS): The system must decide which cell-edge UEs should be assisted by helper UEs and determine which of the D2D connections should be allocated dedicated (intra-cell orthogonal) resources or should reuse cellular resources;

- Resource Block Allocation (RBA): The system must schedule PHY resource blocks (RB) to the D2D and cellular links, taking into account resource reuse decisions of 
the MS step above and measured (reported) interference. In Long Term Evolution systems, for example, such advanced interference measurement schemes are assisted by standardised channel state information reference signals and interference measurement resources [14].

- Power Control (PC): Transmitting nodes, such as the base station and the UEs must set the appropriate transmit power levels.

In this paper we model a multi-cell cellular system in which UEs are willing to provide relaying assistance to cell-edge users and use system simulations to gain insight into the performance of the system when the relaying UE combines amplify-and-forward (AF) or decode-and-forward (DF) type of relaying service with digital or PHY layer network coding (NWC). The key difference between our study and previous studies related to NWC in relay networks is that we take into account the fact that the relaying UEs may have own traffic to send or receive to/from their serving BS. Taking into account this aspect in combination with the interplay between D2D mode selection and the relaying (AF/DF) and NWC scheme allows us to evaluate the system using the same amount of offered traffic independently of the number of nodes operating in relay mode.

\section{SyStem Model AND BASIC CONSIDERATIONS}

\section{A. System Parameters}

We consider a multicell system operating either in a time or frequency division duplex (FDD) mode similarly to a Long Term Evolution Advanced (LTE-A) system operating in FDD spectrum. We assume that signal propagation is affected by both deterministic path-loss and log-normal shadowing, and we denote by $G$ the link gain encompassing both phenomena. Each base station (BS) manages $P$ orthogonal resource blocks (RB) in the uplink (UL) and downlink (DL) directions, that is the total number of RB:s available is $2 P$. In each cell, there are $N$ UEs which establish two-way connections with their respective serving BSs using either a single hop connection in traditional cellular mode or taking advantage of D2D communications to establish a two-hop connection in relay mode. Denote by $M$ the number of required RB:s to establish the $N$ (single or two-hop) end-to-end connections. As we shall see, $M$ depends not only on $N$ and the selected communication modes (i.e. cellular or relay), but also on the type of relaying service provided by the helper UEs (such as UE2 in Figure 1).

The most important notations and system parameters are summarized in Table I.

\section{B. Mode Selection}

Recall from Section I that MS is concerned with assigning relay (helper) UEs to cell edge UEs and to identify the D2D links that should reuse cellular resources. Although mode selection in cellular networks has been studied in the past [15], most MS algorithms focus on single hop D2D communications.
TABLE I

NOTATION AND TERMINOLOGY

\begin{tabular}{|c|c|}
\hline \multicolumn{2}{|r|}{ System Parameters } \\
\hline$N$ & Number of UEs served in each cell \\
\hline$\overline{N_{r}}$ & $\begin{array}{l}\text { Number of UE:s providing relaying service to cell } \\
\text { edge UE:s }\end{array}$ \\
\hline $2 P$ & Number of orthogonal RB:s available (UL+DL) \\
\hline$M$ & $\begin{array}{l}\text { Number of required RB:s to establish the required } N \\
\text { end-to-end connections. In the special case when all } \\
\text { UEs communicate directly with the BS (no relaying): } \\
M=2 N \text {. }\end{array}$ \\
\hline$\rho$ & Path loss decaying exponent. \\
\hline$\sigma_{S}$ & Shadowing parameter expressed in $\mathrm{dB}$. \\
\hline$G$ & $\begin{array}{l}\text { Link gain which takes into account both path loss and } \\
\text { shadowing. }\end{array}$ \\
\hline$G_{1}$ & Path gain between UE1 and the BS \\
\hline$G_{2}$ & Path gain between UE2 and the BS \\
\hline$G_{12}$ & Path gain between UE1 and UE2 \\
\hline$\Delta$ & $\begin{array}{l}\text { Threshold parameter to control the define the cell } \\
\text { edge area }\end{array}$ \\
\hline $\bar{\delta}$ & $\begin{array}{l}\text { Threshold parameter to determine the set of candidate } \\
\text { relay UEs }\end{array}$ \\
\hline $\mathcal{U}_{1}$ & Set of cell edge UEs in a cell, i.e. for which $G_{1}<\Delta$ \\
\hline $\mathcal{U}_{2}$ & $\begin{array}{l}\text { Set of UEs that provide relay service for cell edge } \\
\text { UEs, } N_{r}=\left|\mathcal{U}_{\in}\right|\end{array}$ \\
\hline$\iota\left(\mathcal{U}_{1}\right)$ & Index of the UE in the set $\mathcal{U}_{1}$ that has the lowest $G$ \\
\hline$\kappa\left(\mathcal{U}_{2}, \iota\right)$ & $\begin{array}{l}\text { Index of the UE in the set } \mathcal{U}_{2} \text { for which } \delta_{\min } \text { is the } \\
\text { largest with respect to UE- } \iota \text {, that is the index of the } \\
\text { best relay } \mathrm{UE} \text { in } \mathcal{U}_{2} \text { for } \mathrm{UE}-\iota\end{array}$ \\
\hline $\mathcal{R}$ & $\begin{array}{l}\text { Set of PHY resource block available in each cell; } \\
|\mathcal{R}|=P\end{array}$ \\
\hline$\chi(r)$ & $\begin{array}{l}\text { Measured, estimated or predicted received interfer- } \\
\text { ence power on } \mathrm{RB} r \in \mathcal{R}\end{array}$ \\
\hline$\gamma^{t g t}$ & Target Signal-to-interference-plus-noise ratio (SINR) \\
\hline$\gamma$ & Measured, estimated or predicted SINR \\
\hline$P_{t x, \max }$ & Maximum transmit power for each active link \\
\hline
\end{tabular}

For the coverage extension scenario, we employ the simple heuristic algorithm summarized in Figure 2. As indicated in Table I and Figure 1, the initialization of the sets $\mathcal{U}_{1}$ and $\mathcal{U}_{2},-$ that is the set of cell edge UEs and possible relay UEs - makes use of the threshold parameters $\Delta$ and $\delta$. Simply put, a UE is a cell edge UE if its path gain is less than $\Delta$ and a UE can serve as a relay UE for a cell edge UE if both $G_{12}$ (the UE-relay link) and $G_{2}$ (the relay-BS link) are stronger than $\delta G_{1}$.

If resource reuse between D2D and cellular links is necessary (that is when $M>P$ ), this MS algorithm marks $M-P$ D2D links as being in REUSE mode, otherwise all D2D links are assigned dedicated (i.e. intra-cell orthogonal) resources and get marked as the default DEDI mode. Recall also, that all links between UEs that communicate in cellular mode and the serving BS are always in DEDI mode (that is intracell orthogonality is maintained for cellular links) [3]. Figure 2 also shows how the $(M-P)$ D2D links out of the $M$ D2D links are selected for REUSE mode when $M>P$. 


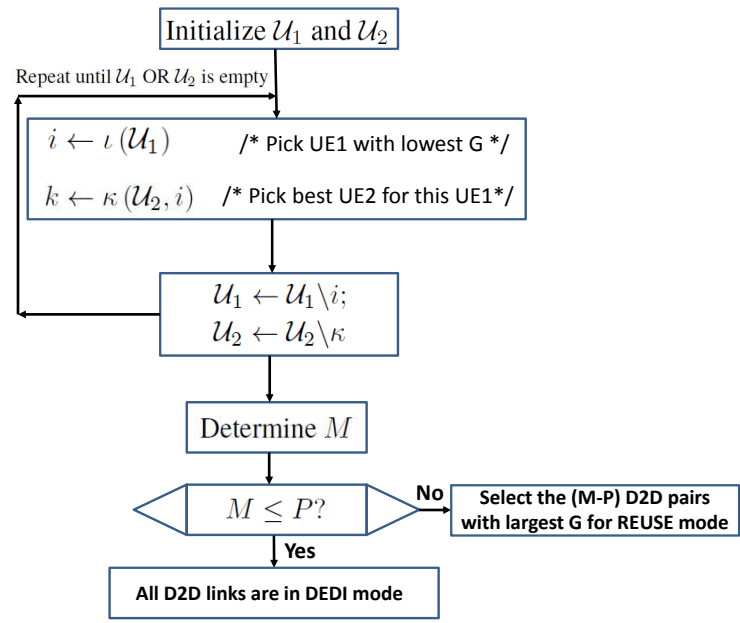

Fig. 2. The task of the mode selection algorithm is to assign relay UEs to cell edge UEs and to mark which D2D links should be assigned orthogonal or reused cellular resources. This marking is then used by the RB assignment (scheduling) algorithm to assign RB:s to both cellular and D2D links.

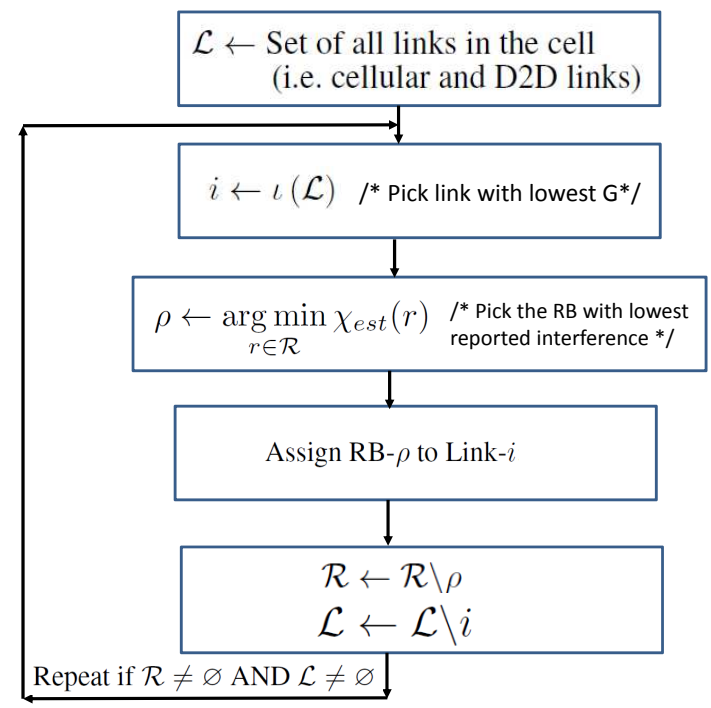

Fig. 3. The task of the mode RB assignment (RBA) algorithm is to assign a RB to each link. Our heuristic RBA algorithm utilizes the interference measurement and reporting mechanism proposed in [16] and supported by the LTE reference signal measurements.

\section{Resource Block Allocation (Scheduling)}

Although resource allocation algorithms applicable for cellular network assisted D2D communications have been proposed in the literature, most of them are not designed for the coverage extension scenario of Figure 1. Therefore, in this paper, we employ the simple heuristic resource allocation algorithm of Figure 3. A key feature of this algorithm is that it reuses cellular resource blocks for D2D communications only when the number of required RB:s $(M)$ is greater than the number of available RB:s $(P)$, in which case the $M-P$ D2D pairs that have the highest path gains (implying that the D2D transmitter and receiver nodes are close to one another) reuse RB:s that are used by the cellular layer. Note that RB assignment to links in REUSE mode involves selecting the link-pairs (e.g. a D2D pair and a cellular link) that should reuse a particular resource block. To this purpose, in this paper we use the MinInterf algorithm proposed by [15].

\section{Power Control}

To better support the D2D communication based range extension solutions, we propose an energy efficient combined link layer and network layer UL PC scheme that can balance the achieved signal-to-interference-plus-noiseratio (SINR) along the two hops of relay assisted connections. The link layer PC method is based on the LTE open loop fractional path loss compensation (OFPC) scheme which is specifically designed for the UL connections, and which was shown to be efficient for underlaid D2D communications [16], [17].

Specifically, the transmit power $P_{t x}$ (in $\mathrm{dB}$ ) is determined as:

$$
P_{t x}=\min \left[P_{t x, \max }, P_{0}-\alpha G\right]
$$

where $\alpha$ is the fractional path loss compensation factor, with $0 \leq \alpha \leq 1$, and $P_{0}$ is a base power level used to control the SINR target which is calculated as:

$$
P_{0}=\alpha\left(\gamma^{t g t}+\chi_{e s t}\right)+(1-\alpha) P_{t x, \max }
$$

where $\chi_{\text {est }}$ represents the measured or estimated noise plus intercell interference power (which is assumed to be perfectly known by the BS). Note that $\alpha=0$ means that each active link transmits with a fixed maximum power $P_{t x, \max }$. In this case, higher path-loss entails lower received power at the receiver (i.e. no path loss compensation). Conversely, with $\alpha=1$ the path-loss is fully compensated by the power control scheme (up to $P_{t x, \max }$ ) and, accordingly, the transmitted power of low path loss links can be reduced compared with high path links, with the aim of achieving the same desired SINR for all active links. The intermediate setting $0<\alpha<1$ entails fractional path loss compensation where a certain degree of SINR unbalance is maintained among different links. As we will see in the numerical section, in the D2D relay assisted coverage extension scenarios, increasing $\alpha$ may lead to overall power reduction, depending on the SNR target, base power level and system geometry.

The network layer power control strategy forces power reduction on one of the two links of two-hop connections without affecting the end-to-end rate. Specifically, let $\gamma_{1}$ and $\gamma_{2}$ denote the estimated SINRs of the two links of a twohop connection, and assume that $\gamma_{1}-\gamma_{2}=\Theta[\mathrm{dB}]$, with $\Theta>0$. In this case, the power of Transmitter $_{1}$ is reduced by $\Theta[\mathrm{dB}]$, in order to balance the expected achieved SINRs of the two links. The rationale for this network layer scheme is that the end-to-end performance is determined by the bottleneck (lowest rate) link, and, hence, the SINR of the other link can be reduced without affecting the end-to-end rate. It follows that it is necessary the the link layer PC is carried out on both links of the two-hops connection. On the other hand, as it will be discussed in the following, the use of relaying might 
require the allocation of a mixture of UL and DL resources to two-hops connections. Hence, we assume that the same OFPC power control described above is implemented for all links, irrespective of whether they use UL or DL resources.

\section{DECODE AND ForWARD (DF) RELAYING Without AND WITH NETWORK CODING}

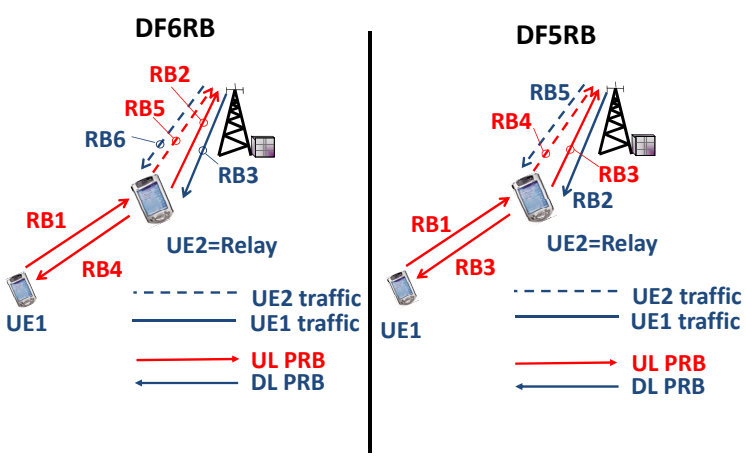

Fig. 4. Decode and forward relaying without (left) and with (right) network coding. In the simple example of this figure, using the notation of Table I $N=2$ and $N_{r}=1$. Without NWC (left), the number of required RB:s is $M=N+N_{r}=3$ in both the UL and DL. With NWC (right), $M=N+N_{r}$ in the UL but only $M=N$ in the DL.

Figure 4 shows the operation of a decode and forward (DF) relay assisted communication scenario in which the helper UE (UE2) provides relaying service to the cell edge UE (UE1) in addition to communicating (i.e. transmitting and receiving) its own traffic with the BS. Without network coding (left hand side of Figure 4), 6 RB:s are needed to support the UE1-BS and the UE2-BS two-way connections. In general, the required resource blocks to support both the UE1-BS and UE2-BS connections, the number of required RB:s is $M=N+N_{r}$ in the uplink as well as in the downlink, where $N_{r}$ is the number of UE:s providing relaying service to cell edge UE:s, as indicated in Table I.

An important consequence of the arrangement of the DF scenario without NWC is that the RB:s used for D2D communications (e.g. RB1 and RB4 on the left and RB1 and RB3 on the right) can be reused by the cellular layer (that is other cellular UEs not shown in the figure).

DF with network coding enables UE2 to employ joint cellular and D2D transmission using a single resource (RB3 in Figure 4 (right)) provided that UE1 can receive and decode signals in the uplink. This implies that the required RB:s in the DL is $M=N$ (rather than $N+N_{r}$ as in the case without NWC). Note that using RB3 for the transmission of NWC data implies that RB3 cannot be reused by other cellular UEs (not shown in the Figure).

\section{AMPLify AND ForWARd (AF) RELAYing With NETWORK CODING}

Figure 5 shows the operation of the amplify and forward (AF) relay assisted communication scenario in which the helper UE (UE2) provides relaying service to UE1 in addition to communicating its own traffic with the BS. Similarly to the

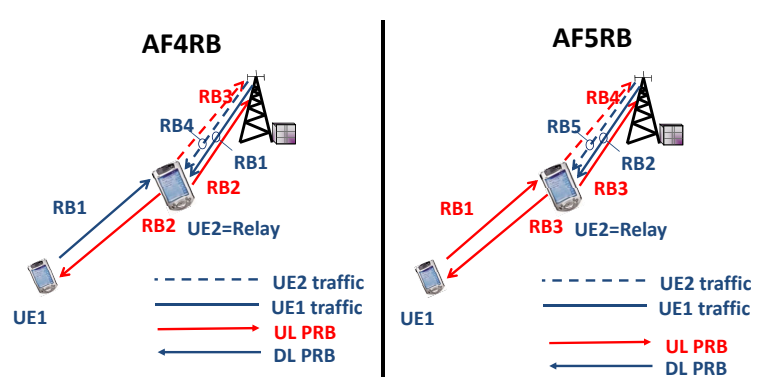

Fig. 5. Analogue network coding using the two-RB and three-RB NWC schemes similarly to the two-slot and three-slot schemes proposed by [18] and [19]. In the simple example of this figure, using the notation of Table I $N=2$ and $N_{r}=1$. In the two-RB analogue NWC scehem (left), the number of required RB:s is $M=N$ in both the UL and DL. Using the three-RB analogue NWC scheme (right), $M=N+N_{r}$ UL and $M=N$ DL resources are required.

two-slot and three-slot PHY layer network coding schemes, we distinguish the two-RB and three-RB AF relaying methods.

The two-RB AF scheme (AF4RB, left hand side of Figure $5)$ enables the BS and UE1 to use the same resource (RB1) to transmit to the relay node (UE2) rather than requiring orthogonal resources. For example, as shown in Figure 5, the $\mathrm{BS}$ and UE1 can use the same DL resource (RB1) provided that UE1 can transmit in the DL. Thus, such an arrangement is no longer transparent to UE1, but offers the possibility of efficiently reusing RB:s in the system. Also, a consequence of the two-RB AF scheme is that the simultaneous reception of the two DL signals on RB1 allows the UE2 to transmit the superposition of the two signals to UE1 and the BS using a single resource (RB3) [18], [19] requiring only two RB:s for the two-way communication as illustrated in Figure 5. (Justifying the terminology of the two-RB scheme.) That is in this case the total required number of RB:s is $M=N$ both in the UL and DL direction. On the other hand, the resources used for the D2D link (RB1 and RB2) cannot be reused by other UEs of the cellular layer, since these resources are already reused by the UE2-BS cellular communication.

In the three-RB AF scenario (AF5RB, Figure 5, right), the UE1 and the BS transmit their respective packets to UE2 using orthogonal resource RB1 (uplink) and RB2 (downlink) respectively. In the arrangement of Figure 5 the UE2 uses an UL resource to transmit the network coded packet to UE1 and the BS and thus the number of required UL RB:s is $M=N+N_{r}$ while the number of required DL RB:s is $M=N$. Note that in this three-RB scheme, one RB (RB1) can be reused by other cellular UEs of the cellular layer (not shown in the Figure), since RB1 is only used by the D2D link.

\section{Calculating the End-TO-End RATES IN The DF AND AF SCENARIOS}

We assume that each link is affected by white noise plus interference by transmissions ongoing over the same RB, either from a node in the same cell reusing the same resource (intra-cell interference), or from nodes in adjacent cells (intercell interference). We also assume that the interference 
matches the white noise-like model, i.e., the SINR for each link is evaluated as $\gamma=\frac{P_{r x}}{\chi}$, where $\chi$ is the overall interference term, encompassing both noise and interference, and $P_{r x}$ is the received power.

Finally, in this paper we assume ideal link level rate adaptation at each terminal, i.e.:

$$
R=W \log _{2}(1+\gamma)
$$

where $W$ is the system bandwidth.

\section{A. Decode-and-Forward}

For the end-to-end rate calculations for the two-hop connections in the DF case (UE1-UE2-BS and BS-UE2-UE1), we make the important assumption that a continuous flow of packets is maintained yielding an end-to-end rate of $R=$ $\min \left[R_{1}, R_{2}\right]$, where $R_{1}$ and $R_{2}$ are the achievable rates in the first and second hops respectively.

\section{B. Amplify-and-Forward}

In order to express the end-to-end rates in the AF case, we refer to the notations defined in Table II.

TABLE II

NOTATIONS

\begin{tabular}{|c|l|}
\hline \hline \multicolumn{2}{|c|}{ Notations for the AF case } \\
\hline$P_{A}, P_{B}, P_{R}$ & $\begin{array}{l}\text { Transmitting powers of } U E_{1}, \mathrm{BS}, \text { and } U E_{2}, \\
\text { respectively. }\end{array}$ \\
\hline$G_{A, R}, G_{B, R}$ & $\begin{array}{l}\text { Gains of links } U E_{1}-U E_{2} \text { and BS-UE } \\
\text { tively. }\end{array}$ \\
\hline$G_{R, A}, G_{R, B}, \begin{array}{l}\text { Gains of links } U E_{2}-U E_{1} \text { and } U E_{2} \text {-BS, respec- } \\
\text { tively. }\end{array}$ \\
\hline$\chi_{A}, \chi_{B}, \chi_{R}$ & $\begin{array}{l}\text { Noise plus interference term at nodes } U E_{1}, U E_{2}, \\
\text { BS, respectively. }\end{array}$ \\
\hline$\gamma_{A}$ and $\gamma_{B}$ & $\begin{array}{l}\text { End-to-end equivalent SINRs for uplink and } \\
\text { downlink connections, respectively. }\end{array}$ \\
\hline
\end{tabular}

In the AF4RB scenario (Figure 5, left), under the assumption that the end receiver may fully cancel the interference (this is possible in the case of perfect channel knowledge at the transmitter), $\gamma_{A}$ and $\gamma_{B}$ are determined as in [18], i.e.:

$$
\begin{aligned}
\gamma_{A} & =\frac{P_{R} G_{R, A}}{\chi_{A}\left(1+\frac{P_{A} G_{A, R}+\chi_{R}}{P_{B} G_{B}, R}\right)+\chi_{R} \frac{P_{R} G_{R, A}}{P_{B} G_{B, R}}} \\
\gamma_{B} & =\frac{P_{R} G_{R, B}}{\chi_{B}\left(1+\frac{P_{B} G_{B, R}+\chi_{R}}{P_{A} G_{A, R}}\right)+\chi_{R} \frac{P_{R} G_{R, B}}{P_{A} G_{A, R}}}
\end{aligned}
$$

It is worth noting that such equivalent end-to-end SINRs are lower than the single link SINRs $\frac{P_{R} G_{R, A}}{\chi_{A}}$ and $\frac{P_{R} G_{R, B}}{\chi_{B}}$, respectively. The reason for this is twofold: $(i)$ the interference plus noise term of the first connection is amplified and retransmitted, so that the performance are affected by $\chi_{R}$; (ii) interference cancelation at the receiver reduces the effective received power, that is like increasing the effective noises $\chi_{A}$ and $\chi_{B}$.

Recall that in the AF5RB case ((Figure 5, right)) the $U E_{1}$ and the BS transmit their packets to the relay using two orthogonal RBs. Hence, the relay forwards a weighted sum of the received signals, with weights $\alpha_{A}$ and $\alpha_{B}$, respectively, with $\alpha_{A}^{2}+\alpha_{B}^{2}=1$, as it was proposed in [18]. Let now denote by $\chi_{A, R}$ and $\chi_{B, R}$ the noise plus interference terms at node $U E_{2}$ on the RB assigned to $U E_{1}-U E_{2}$ and BS- $U E_{1}$ connections, respectively. Then, using the same notations as before, and assuming again perfect interference cancelation at the receivers, we can derive the end-to-end equivalent SINRs for uplink and downlink connections as:

$$
\begin{aligned}
\gamma_{A} & =\frac{P_{R} G_{R, A}}{\chi_{A}\left(1+\frac{\alpha_{A}^{2} P_{A} G_{A, R}+\alpha_{A}^{2} \chi_{A, R}+\alpha_{B}^{2} \chi_{B, R}}{\alpha_{B}^{2} P_{B} G_{B} R}\right)+\eta_{A, R}+\eta_{B, R}}{ }_{P_{R} G_{R, B}}=\frac{\chi_{B}\left(1+\frac{\alpha_{B}^{2} P_{B} G_{B, R}+\alpha_{A}^{2} \chi_{A, R}+\alpha_{B}^{2} \chi_{B, R}}{\alpha_{A}^{2} P_{A} G_{A, R}}\right)+\psi_{A, R}+\psi B, R}{\chi_{B}},
\end{aligned}
$$

where we introduced the following notations:

$$
\begin{aligned}
\eta_{A, R} & \triangleq \chi_{A, R} \frac{\alpha_{A}^{2} P_{R} G_{R, A}}{\alpha_{B}^{2} P_{B} G_{B, R}} \\
\eta_{B, R} & \triangleq \chi_{B, R} \frac{P_{R} G_{R, A}}{P_{B} G_{B, R}} \\
\psi_{A, R} & \triangleq \chi_{A, R} \frac{P_{R} G_{R, B}}{P_{A} G_{A, R}} \\
\psi_{B, R} & \triangleq \chi_{B, R} \frac{\alpha_{B}^{2} P_{R} G_{R, B}}{\alpha_{A}^{2} P_{A} G_{A, R}} .
\end{aligned}
$$

Note that, similarly as before, the equivalent end-to-end SINRs are lower than the single link SINRs. Moreover, in this case parameters $\alpha_{A}$ and $\alpha_{B}$ affect the final equivalent SINRs. As an example, if we consider $\alpha_{A}=\alpha_{B}=\frac{1}{\sqrt{2}}$, and we assume for simplicity $\chi_{A, R}=\chi_{B, R}=\chi_{R}$ it is straightforward to get:

$$
\begin{aligned}
\gamma_{A} & =\frac{P_{R} G_{R, A}}{\chi_{A}\left(1+\frac{P_{A} G_{A, R}+2 \chi_{R}}{P_{B} G_{B}, R}\right)+2 \chi_{R} \frac{P_{R} G_{R, A}}{P_{B} G_{B, R}}} \\
\gamma_{B} & =\frac{P_{R} G_{R, B}}{\chi_{B}\left(1+\frac{P_{B} G_{B, R}+2 \chi_{R}}{P_{A} G_{A, R}}\right)+2 \chi_{R} \frac{P_{R} G_{R, B}}{P_{A} G_{A, R}}}
\end{aligned}
$$

i.e., the effect of the noise at the intermediate node is twice than in the $A F 4 R B$ case. The rationale for this impairment is that in this case at the intermediate node the contribution of two independent noises plus interference terms are summed. Of course, by properly choosing $\alpha_{A}$ and $\alpha_{B}$ we can obtain higher SINRs. In particular, we adopt the same strategy proposed in [18], that is we choose $\alpha_{A}$ and $\alpha_{B}$ which minimize the sum-BER at high SNR for a fixed total source power. That is, more relay power is allocated to the signal received from the source with the largest transmit power.

\section{NUMERICAL RESULTS}

\section{A. Simulation Setup}

We implemented the DF (without and with network coding) and the AF (with PHY layer network coding) D2D communications enabled relaying strategies in the RUdimentary NEtwork (RUNE) simulator [20] to evaluate their performance at the system level. Recall that in our work, the relaying UEs have own DL and UL traffic in addition to providing the relaying service.

RUNE allows to generate an environment with $C$ hexagonal cells of a radius of $R$ and $N$ UEs in each cell that are 
deployed randomly within the cell with a distance from the BS uniformly distributed in the range $[0, R]$. In each such Monte Carlo experiment, mode selection (MS) and resource block allocation (RBA) are executed according to the heuristic algorithms of Figure 2 and Figure 3. As indicated in Figure 2, the MS algorithm needs to evaluate the number of required RBs, which in turn depends on the relaying strategy, as described in Figure 4 and Figure 5.

To evaluate the SINR and rate performance, we consider a fixed number of $N_{i t}>1$ iterations, where at each iteration the cells are processed in a sequential manner, following a predetermined order. Recall from Figure 3, the measured interference $\chi_{\text {est }}(r)$ is a required input to the RBA algorithm. Therefore, each receiver measures the received interference power on each RB. We assume that the measured interference at the receiver is reported to its associated transmitter node and that each transmitter uses the LTE open loop fractional path loss compensating power control algorithm with path loss compensation factor $\alpha$ to determine its transmit power [17], [16].

Note that executing $N_{i t}>1$ iterations approximates a real network operation, in which resource assignments are executed by each BS without intercell coordination, so that interference in each cell depends on the resource allocation decisions made in neighboring cells. In our simulations, after $N_{i t}$ iterations of resource allocation, each cell is affected on average by similar interference power levels appropriately modeling a real multicell system. As for data traffic, we assume full (saturated) buffers at each transmitter. Table III summarizes the main simulation parameters.

TABLE III

SIMULATION PARAMETERS

\begin{tabular}{|c|l|}
\hline \hline \multicolumn{2}{|c|}{ General simulation parameters } \\
\hline R (Cell Radius) & $500 \mathrm{~m}$ \\
\hline System bandwidth $W$ & $5 \mathrm{MHz}$ \\
\hline Carrier frequency & $2 \mathrm{GHz}$ \\
\hline Maximum TX Power $P_{t x, \max }$ & $250 \mathrm{~mW}$ \\
\hline Number of RBs assigned to each user & 1 \\
\hline Total available RBs per cell $(2 P)$ & 60 \\
\hline Propagation specific Parameters \\
\hline Noise power & $-174 \mathrm{dBm}$ \\
\hline BS antenna height & $15 \mathrm{~m}$ \\
\hline UE antenna height & $1.8 \mathrm{~m}$ \\
\hline Path loss decaying exponent $(\rho)$ & 4 \\
\hline $\begin{array}{c}\text { Log-normal Shadowing } \\
\text { standard deviation }(\sigma S)\end{array}$ & $10 \mathrm{~dB}$ \\
\hline Simulation Algorithm's parameters \\
\hline Number of mobile users per cell $(N)$ & 25 \\
\hline MS relative threshold values $(\delta)$ & $7 \ldots 16[\mathrm{~dB}]$ \\
\hline Pathloss compensation factor $(\alpha)$ & $0 \ldots 1$ \\
\hline $\begin{array}{c}\text { Number of simulations for a given value } \\
\text { of } \alpha \text { and } \delta\end{array}$ & 100 \\
\hline SINR target $\left(\gamma^{t g t}\right)$ & $7 \mathrm{~dB}$ \\
\hline Number of iterations for the SA $\left(N_{i t}\right)$ & 3 \\
\hline \hline
\end{tabular}

\section{B. Numerical Results}

In this section we are primarily interested of the achievable gains in terms of average power consumption reduction and rate improvements for cell edge UEs when employing the proposed DF and AF relaying and resource allocatio strategies without/with employing network coding. Since coverage is typically more problematic in the UL direction, we focus on the performance of UL transmissions as a function of the path loss compensation factor $\alpha$ and the $\delta$ parameter that controls relay selection.

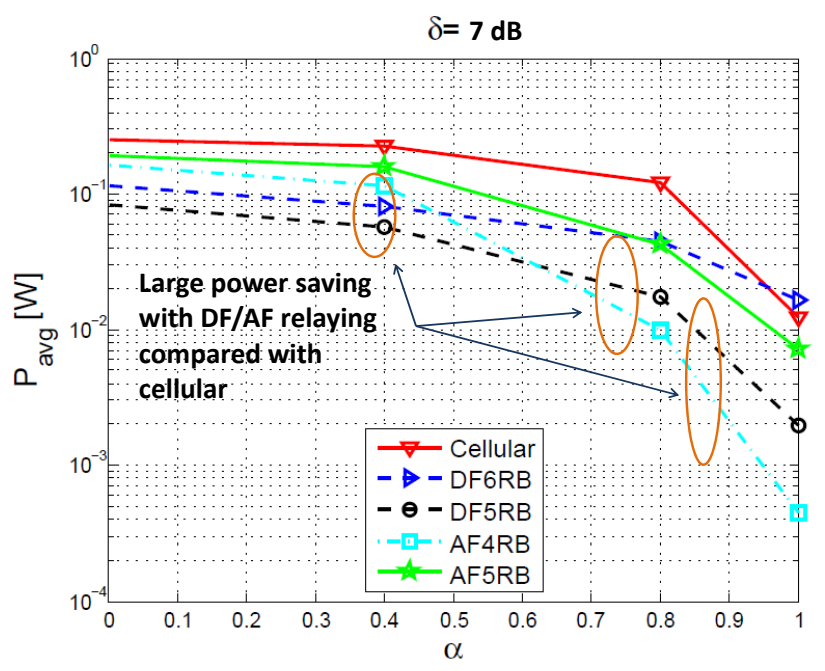

Fig. 6. Average power consumption of cell edge UEs as a function of the path loss compensation factor with $\delta=7 \mathrm{~dB}$.

Figure 6 compares the average power consumption of cell edge UEs when employing cellular (single hop) or D2D relay assisted schemes (DF6RB, DF5RB, AF4RB, AF5RB). Note that higher $\alpha$, implies that higher power saving can be achieved by all four relaying schemes with respect to the cellular case. This gain is due to fact that the link quality of cell edge UEs can be noticeably increased by allowing two-hops connections. Note also that a certain level of power saving is achieved in the case $\alpha=0$. This is due to presence of the network layer power control described in Section II-D. Although all four relaying schemes reduce the average power consumption, it is clear that from a power saving perspective, DF with network coding (DF5RB) and the 2-RB AF scheme (AF4RB) are the most advantageous. The intuitive explanation is that AF4RB avoids resource reuse within a cell and therefore UEs employing the AF4RB scheme do not cause intracell interference.

Figure 7 shows the average power consumption when $\delta=16 \mathrm{~dB}$. Recall that a higher value of $\delta$ implies that only UEs with much stronger links towards both the cell edge UE and the BS can become relaying UEs as compared with the path gain of the link between the celle edge UE and the BS. Therefore, with $\delta=16 \mathrm{~dB}-$ compared with the $\delta=7$ $\mathrm{dB}$ case of Figure $6-$, the path gains of the two hops of relay assisted connections are more balanced, since both path gains must be substantially stronger than the single hop gain. We notice in Figure 7 that the average power consumption 


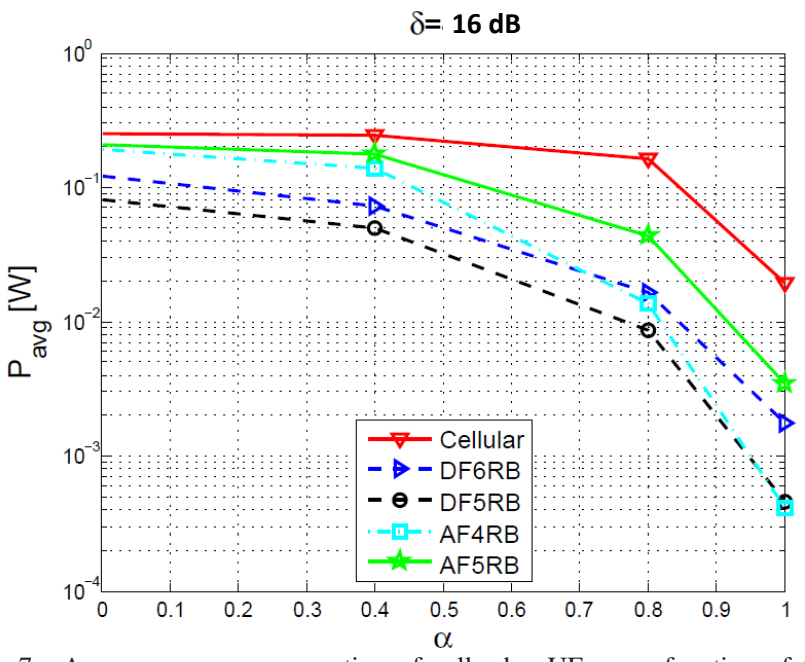

Fig. 7. Average power consumption of cell edge UEs as a function of the path loss compensation factor with $\delta=16 \mathrm{~dB}$.

of the AF4RB scheme is only slightly reduced with respect to Figure 7, whereas the power consumption of the other relaying schemes substantially decreases. For example, the average power consumption of cell edge UEs with $\alpha=1$ with the DF5RB scheme is around $2 \mathrm{~mW}(3 \mathrm{dBm})$ with $\delta=7$ $\mathrm{dB}$ and only $0.4 \mathrm{~mW}(-4 \mathrm{dBm})$ with $\delta=16 \mathrm{~dB}$.

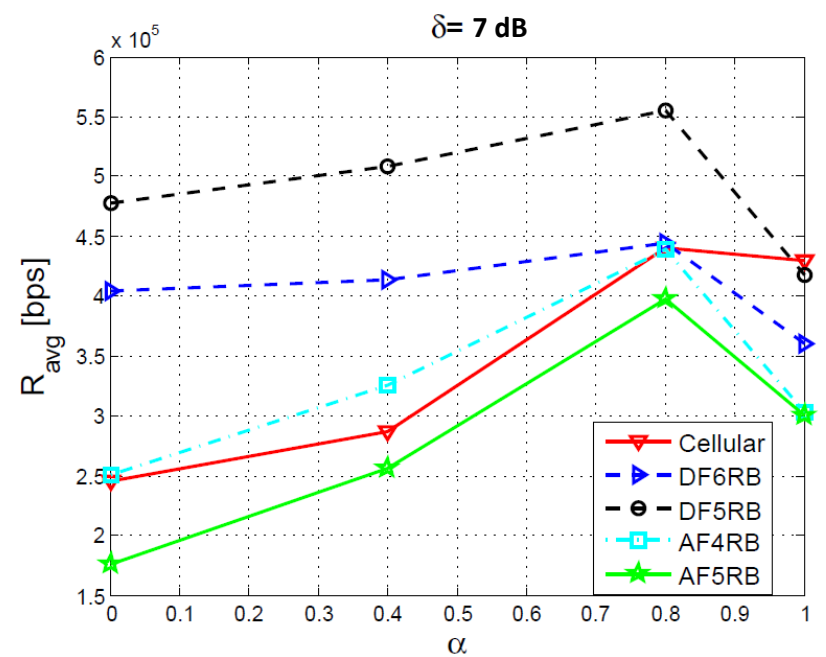

Fig. 8. Average rate in the UL direction of cell edge UEs as a function of the path loss compensation factor with $\delta=7 \mathrm{~dB}$.

Figures 8 and 9 compare the achieved UL end-to-end rates of the cell edge UEs as the function of the path loss compensation factor with $\delta=7 \mathrm{~dB}$ and $\delta=16 \mathrm{~dB}$, respectively. As expected, the DF relaying strategy employing network coding (DF5RB) provides the best performance both with $\delta=7 \mathrm{~dB}$ and $\delta=16 \mathrm{~dB}$. As Figure 9 illustrates, the DF schemes outperform the AF schemes due to the negative impact of amplifying the noise in the AF schemes.

Recall that in the D2D relaying schemes, the relaying UEs have own traffic to transmit to the BS and it is therefore important to examine the performance of all UEs rather than

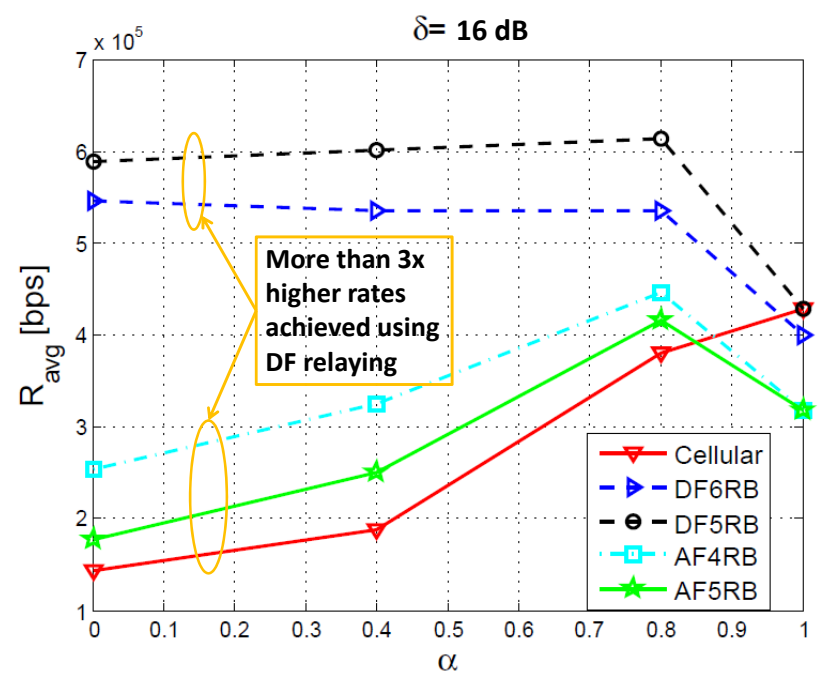

Fig. 9. Average rate in the UL direction of cell edge UEs as a function of the path loss compensation factor with $\delta=16 \mathrm{~dB}$.

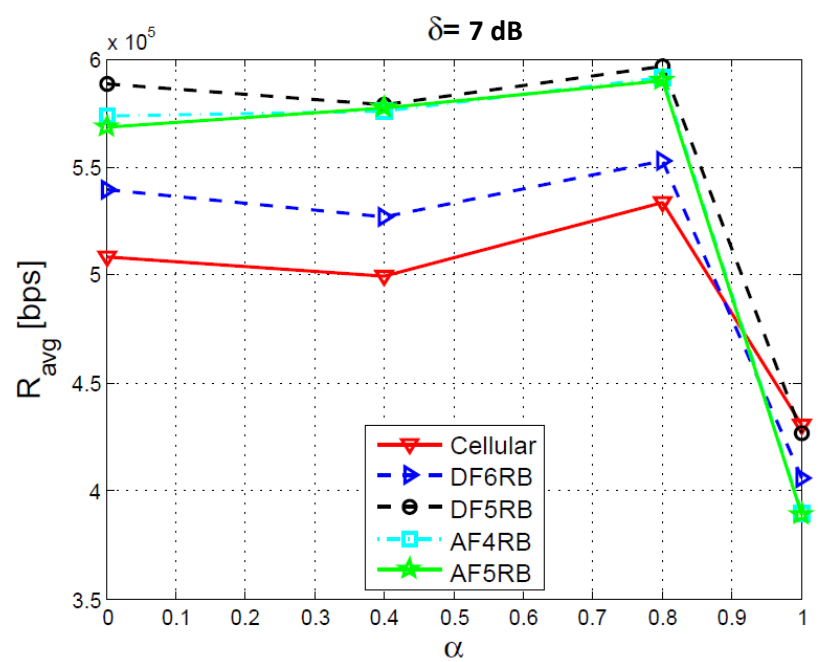

Fig. 10. Average rate in the UL direction of all UEs (cell edge as well as relaying UEs) as a function of the path loss compensation factor with $\delta=7$ dB.

focusing on the cell edge UEs only. Figure 10 shows the rate performance of all UEs in the UL direction, with the setting of $\delta=7 \mathrm{~dB}$. Again, DF5RB achieves the highest end-to-end rates, especially with moderate path loss compensation.

Figure 11 evaluates the impact of D2D based relaying on the Jain fairness index as a function of $\alpha$. It is clear that relaying techniques achieve higher values of the fairness index compared with traditional cellular transmission. The DF techniques offer better resource sharing fairness compared with AF, in particular DF5RB offers the highest fairness.

Finally, Figures 12-13 compare the DL rate performance of the D2D relaying schemes with $\delta=7$ and $\delta=16 \mathrm{~dB}$. We again observe that DF5RB scheme offers superior performance due to a combination of efficient resource usage and digital network coding. The improvement over the achievable cellular rate depends on the path loss compensation factor $\alpha$ and we can see that the best rate performance can be achieved by 


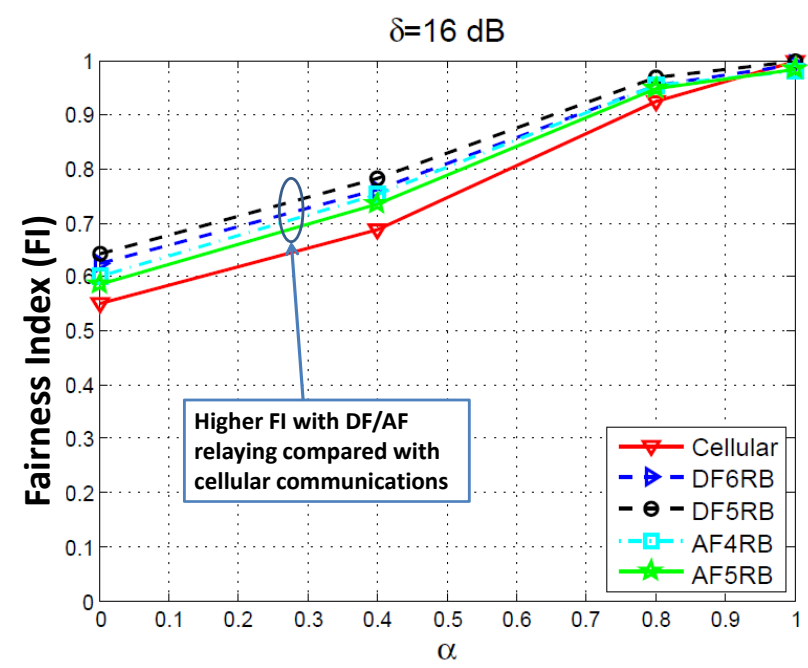

Fig. 11. Fairness index (FI) of UEs regarding the achieved UL transmit rates as a function of the path loss compensation factor with $\delta=16 \mathrm{~dB}$.

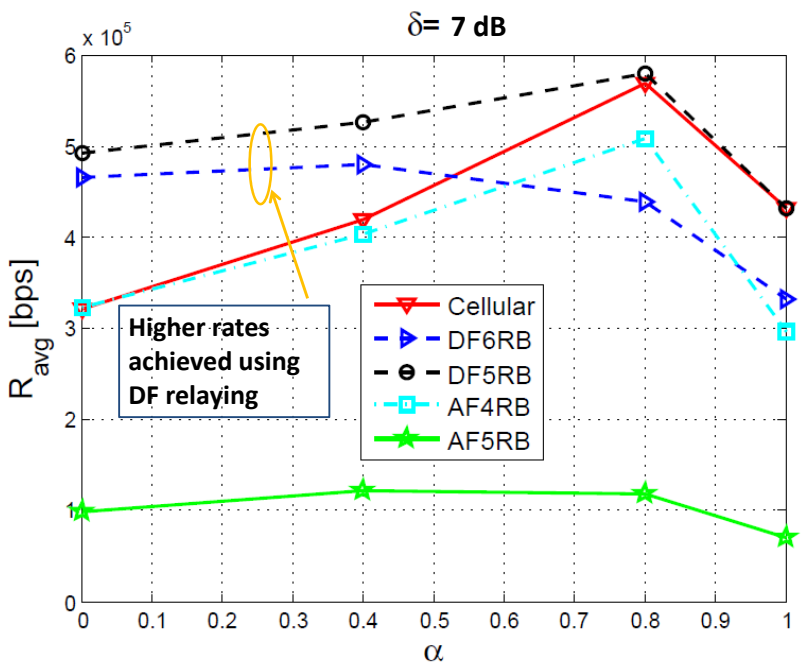

Fig. 12. DL rate performance of cell edge UEs as a function of the $\alpha$ path loss compensation factor. DF5RB provides superior performance, although the rate improvement over the achievable cellular rate is negligible when $\alpha$ is set appropriately.

setting $\delta$ to a relatively high value $(\delta=16 \mathrm{~dB})$.

\section{CONCLUSIONS}

In this paper we used system simulation to examine the performance of D2D communications enabled relaying schemes when combined with digital or PHY layer network coding. When employing decode-and-forward and amplify-andforward schemes, appropriate resource allocation algorithms must be developed, since when using D2D based relaying, the relaying UEs themselves have own traffic that must be properly multiplexed with the relayed traffic. To this end, we proposed heuristic algorithms for mode selection and RB allocation applicable in relaying schemes using AF or DF relaying strategies. We found that the trade off between power consumption and achieved rate can be effectively addressed by relaying schemes, and especially the DF scheme with network

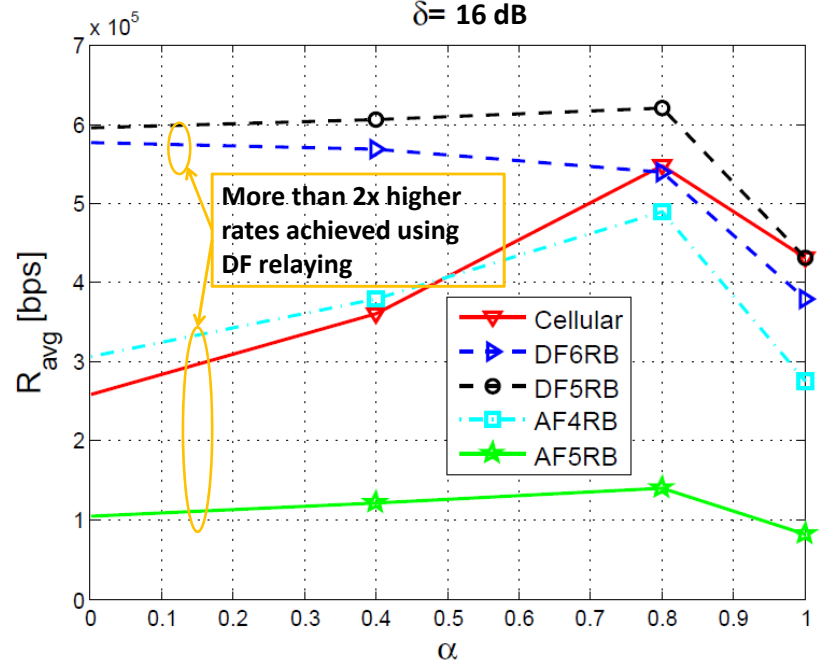

Fig. 13. DL rate performance of cell edge UEs as a function of the $\alpha$ path loss compensation factor. DF5RB provides superior performance, and in this case $(\delta=16 \mathrm{~dB})$ the rate improvement over cellular transmission is higher than with $\delta=7 \mathrm{~dB}$.

coding can largely improve the performance of cell edge UEs. We also found that tuning the relay selection parameter $\delta$ and the path loss compensation factor $\alpha$ also greatly affects the overall system performance including the achieved fairness.

\section{REFERENCES}

[1] H. Wu, C. Qiao, S. De, and O. Tonguz, "Integrated Cellular and Ad Hoc Relaying Systems:iCAR," IEEE J. on Selected Areas in Communications, vol. 19, no. 10, pp. 2105-2115, 2001.

[2] F. H. P. Fitzek, M. Katz, and Q. Zhang, "Cellular controlled short-range communications for cooperative $\mathrm{p} 2 \mathrm{p}$ networking," Wireless Personal Communications, vol. 48, no. 1, pp. 141-155, January 2009, dOI: 10.1007/s11277-007-9425-6.

[3] G. Fodor, E. Dahlman, G. Mildh, S. Parkvall, N. Reider, G. Mikls, and Z. Turnyi, "Design Aspects of Network Assisted Device-to-Device Communications," IEEE Comm. Mag., vol. 50, pp. 170-177, 2012.

[4] S. Wen, X. Zhu, Y. Lin, Z. Lin, X. Zhang, and D. Yang, "Achievable Transmission Capacity of Relay-Assisted Device-to-Device (D2D) Communication Underlay Cellular Networks," in Proc. of the IEEE Vehic. Tech. Conf. (VTC), Sep. 2013.

[5] A. Asadi, Q. Wang, and V. Mancuso, "A Survey on Device-to-Device Communication in Cellular Networks," arXiv, Oct. 2013. [Online]. Available: http://arxiv.org/abs/1310.0720

[6] K. Doppler, M. Rinne, C. Wijting, C. B. Riberio, and K. Hugl, "D2D Communications Underlaying an LTE Cellular Network," IEEE Comm. Mag., vol. 7, no. 12, pp. 42-49, December 2009.

[7] B. Zafar, S. Gherekhloo, and M. Haardt, "Analysis of Multihop Relaying Networks,” IEEE Veh. Tech. Mag., vol. 7, no. 3, pp. 40-47, August 2012.

[8] L. Chen, Y. Huang, F. Xie, Y. Gao, L. Chu, H. He, Y. Li, F. Liang, and Y. Yuan, "Mobile Relay in LTE-Advanced Systems," IEEE Comm. Mag., vol. 51, no. 11, pp. 144-151, Nov. 2013.

[9] J. Prez-Romero and R. A. O. Sallent, "Enhancing Cellular Coverage through Opportunistic Networks with Learning Mechanisms," in Proc. of the IEEE Global Telecommun. Conf., Atlanta, GA, USA, Dec. 2013.

[10] L. Wei, R. Q. Hu, Y. Qian, and G. Wu, "Energy-Efficiency and Spectrum-Efficiency of Multi-Hop Device-to-Device Communications Underlaying Cellular Networks," IEEE Transactions on Vehicular Technology, 2015, dOI 10.1109/TVT.2015.2389823.

[11] Q. Guo and B. Zhang, "Device-to-Device Uplink Relay Underlaying Cellular Networks for Multiple Discrete Access," Journal of Computational Information Systems, vol. 11, no. 2, pp. 711-718, January 2015, dOI $10.12733 /$ jcis 13145 . 
[12] J. M. B. da Silva Jr, G. Fodor, and T. F. Maciel, "Performance Analysis of Network-Assisted Two-Hop D2D Communications," IEEE Broadband Wireless Access Workshop, vol. Austin, TX, USA, December 2014.

[13] G. Fodor, S. Parkvall, S. Sorrentino, P. Wallentin, Q. Lu, and N. Brahmi, "Device-to-Device Communications for National Security and Public Safety," IEEE Access, vol. 2, pp. 1510-1520, January 2015.

[14] W. Xi, X. Yun, S. Nagata, Y. Kishiyama, and L. Chen, "An Enhanced Interference Measurement Scheme for CoMP in LTE-Advanced Donwlink," in IEEE ICC Signal Processing for Communications Symposium, Budapest, Hungary, June 2013, pp. 4870-4874.

[15] M. Belleschi, G. Fodor, and A. Abrardo, "Performance Analysis of a Distributed Resource Allocation Scheme for D2D Communications," in IEEE Workshop on Machine-to-Machine Communications. IEEE, 2011.

[16] M. Belleschi, G. Fodor, D. D. Penda, A. Pradini, M. Johansson, and A. Abrardo, "Benchmarking Practical RRM Algorithms for D2D Communications in LTE Advanced," Wireless Personal Communications (Springer), 2014.

[17] G. Fodor, M. Belleschi, D. D. Penda, M. Johansson, and A. Abrardo, "A Comparative Study of Power Control Approaches for D2D Communications," in IEEE ICC, Budapest, June 2013.

[18] R. H. Y. Louie, Y. Li, and B. Vucetic, "Practical Physical Layer Network Coding for Two-Way Relay Channels: Performance Analysis and Comparison," IEEE Transactions on Wireless Communications, vol. 9, no. 2, pp. 764-777, 2010.

[19] F. Yang, M. Huang, S. Zhang, and W. Zhou, "Performance Analysis on Two-Way Relay System with Co-Channel Interference," Wireless Personal Communications, vol. 72, no. 1, pp. 415-434, 2013. [Online]. Available: http://dx.doi.org/10.1007/s11277-013-1021-3

[20] J. Zander, S.-L. Kim, M. Almgren, and O. Queseth, Radio Resource Management for Wireless Networks. Artech House, 2001. 